\title{
Structural and Optical Properties of Antimony-Containing Epitaxial Layers Grown on GaSb by MOCVD
}

\author{
M. WesolowsKi* AND W. Strupiński \\ Institute of Electronic Materials Technology, Wólczyńska 133, 01-919 Warsaw, Poland
}

\begin{abstract}
Experimental results on MOCVD epitaxy of some antimonides on GaSb substrates are presented. Specific technological problems, which effect in narrow window of process parameters, were overcome and good quality of $\mathrm{GaSb} / \mathrm{GaSb}$, InGaSb/GaSb and InGaAsSb/GaSb layers was obtained. Structural, optical and electrical characterisation data are shown and discussed. Developed technology can state a ground work for realisation of antimonide-based optoelectronic devices.
\end{abstract}

PACS numbers: 81.15.Gh, 81.05.Ea, 68.55.A-, 68.55.ag, 68.55.J-, 68.55.Nq

\section{Introduction}

Antimonides become important semiconductor family. Several sorts of infrared photodetectors and lasers, photovoltaic cells and other electronic devices are already established or will be developed in the near future [1]. Beside molecular beam epitaxy (MBE), the metalorganic chemical vapor deposition (MOCVD) is applied as advanced technique of layer deposition for antimonides. The MOCVD has some advantages, if compared to MBE. Among them are the lower single process cost and higher convenience to commercialization. On the other side, MOCVD is touched by several antimonides-specific problems, making it more difficult to apply than it occurs for arsenides or phosphides [2-5]. In the consequence, successful application of MBE epitaxy of antimonides is transferred to the MOCVD epitaxy with troubles and delay. For some antimonide device applications, like for majority of laser structures, attempts of such transfer are currently unpromising.

\section{Experimental}

We used AIXTRON 200 MOCVD reactor with quartz chamber, halogen-heated susceptor and hydrogen as a carrier gas. Tri-methyl-antimony adduct (TMSb) was selected as antimony precursor, $\mathrm{AsH}_{3}$, tri-methyl-gallium adduct (TMGa) and tri-methyl-indium adduct (TMIn) correspondingly as arsenide, gallium and indium precursors. The temperature was in the $500-600^{\circ} \mathrm{C}$ range, pressure in the 100-500 mbar range. The V/III partial pressures ratio was varied from 0.4 to 3 and typical growth rate was about $0.5 \mu \mathrm{m} / \mathrm{h}$. Produced samples were primarily characterised by optical microscopy and atomic force microscopy (AFM). Next, structural and optical

* corresponding author; e-mail: marek.wesolowski@itme.edu.pl characterisation was carried out by XRD, photoluminescence, absorption or photoreflectance spectroscopy. Impurities and dopants were examined by secondary ion mass spectroscopy (SIMS). Structural quality was investigated by transmission electron microscopy (TEM). Characterisation data were employed to provide technology optimisation and clarification of observed issues.

\section{Results}

All growth attempts indicated that development of good layer morphology was highly influenced by substrate selection and preparation. The surface quality was primarily affected by the substrate orientation. Fig. 1 presents optical microscopy images of layers produced in single process on three different substrates. Best results were typically obtained on disoriented GaSb wafers, for example $\langle 100\rangle 6^{\circ}$ to $\langle 111\rangle$. Secondary, the ability of GaSb surface to produce oxides makes difficult to obtain an oxide-free substrate in the reactor, so special de-oxidation procedures had to be worked out.

Optimal process temperature varied from $550^{\circ} \mathrm{C}$ for GaSb layers to $590^{\circ} \mathrm{C}$ for InGaSb and InGaAsSb layers. While layers quality was highly sensitive on reactor temperature, it was moderately sensitive on the pressure. The optimum pressure value was recognized as about 100 mbar. Improvement of process parameters and proper substrate choice resulted in good, often mirror-like surface with satisfying roughness (measured by AFM) for all binary GaSb, ternary InGaSb and quaternary InGaAsSb materials. The AFM images of optimised layers on $\mathrm{GaSb}$ demonstrated $\mathrm{rms}=0.538$ for $\mathrm{GaSb} / \mathrm{GaSb}, \mathrm{rms}=0.558$ for $\mathrm{InGaSb}$ and $\mathrm{rms}=0.580$ for InGaAsSb.

$\mathrm{In}_{x} \mathrm{Ga}_{1-x} \mathrm{Sb} / \mathrm{GaSb}$ structures exhibited well defined $\mathrm{X}$-ray diffraction (XRD) (004) reflex, which enabled to assign indium composition factor. Several rocking curves are shown in Fig. 2. For compositions up to $x=0.25$ surface morphology of $0.2 \mu \mathrm{m}$ thick layers did not show 

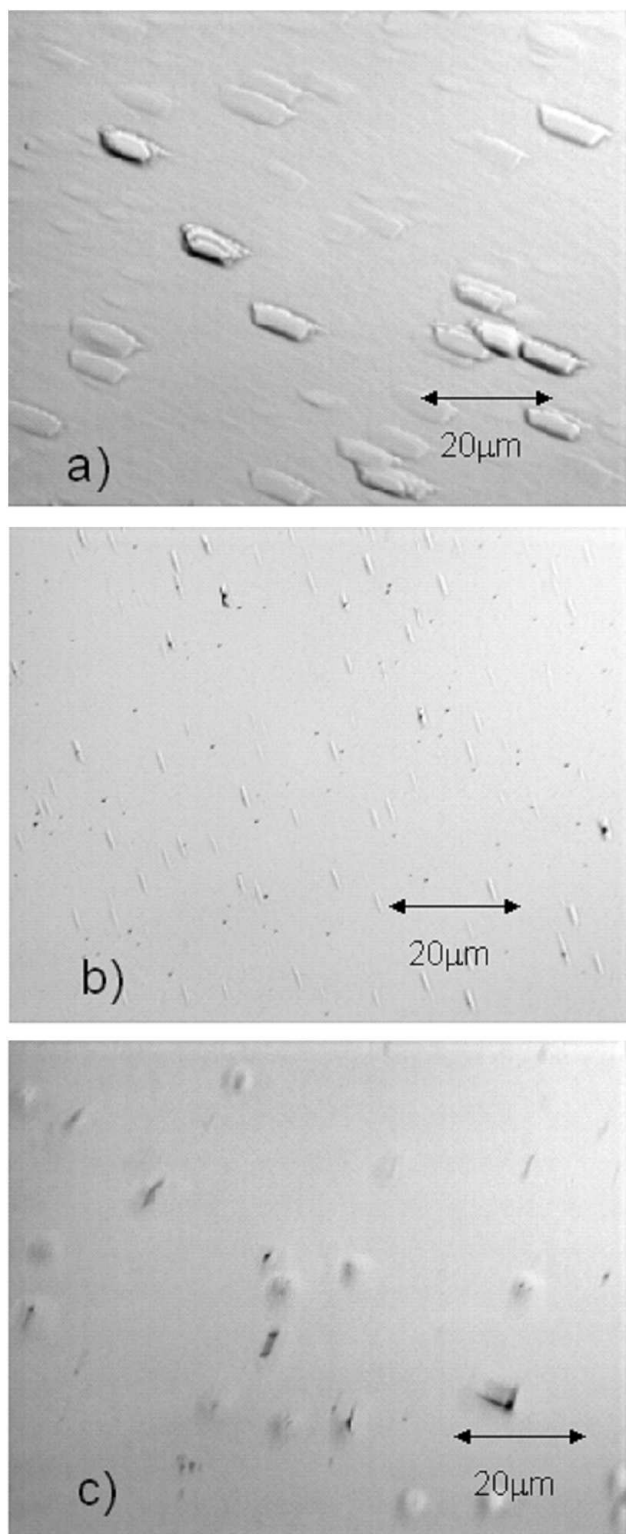

Fig. 1. Surface morphology of InGaAsSb/GaSb layers grown in the same process but on different GaSb substrates: oriented $\langle 110\rangle$ (a), disoriented $\langle 100\rangle 6^{\circ}$ to $\langle 111\rangle$ (b), oriented $\langle 100\rangle$.

dislocation lines. For higher composition factor surface deteriorated, however XRD line was still clearly present until $x$ reached about 0.35 .

The structural quality confirmed by XRD was strongly dependent on $\mathrm{V} / \mathrm{III}$ ratio and on process temperature. The increase of temperature to $590^{\circ} \mathrm{C}$ resulted in improvement of the structure, however better pyrolysis of TMGa in higher temperature induced the abrupt decrease of indium content.

The photoluminescence spectroscopy of InGaSb layers exhibited band-edge emission with transition energy varying with indium composition, as shown in Fig. 3.

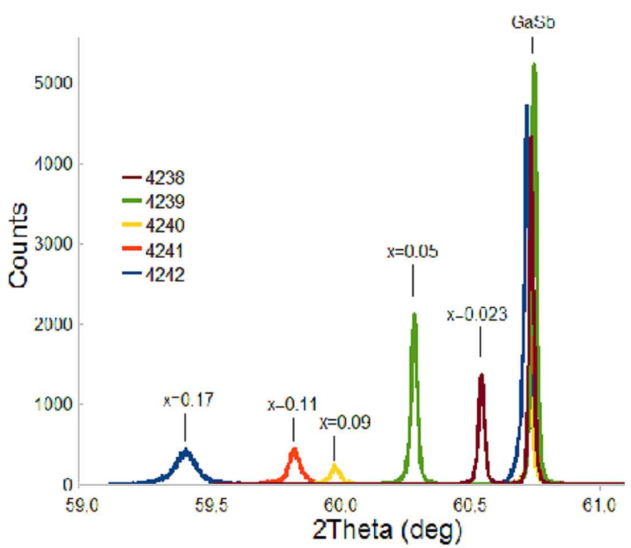

Fig. 2. XRD (004) reflex for $\operatorname{In}_{x} \mathrm{Ga}_{1-x} \mathrm{Sb} / \mathrm{GaSb}$ layers with different compositions, from $x=0$ to $x=0.17$.

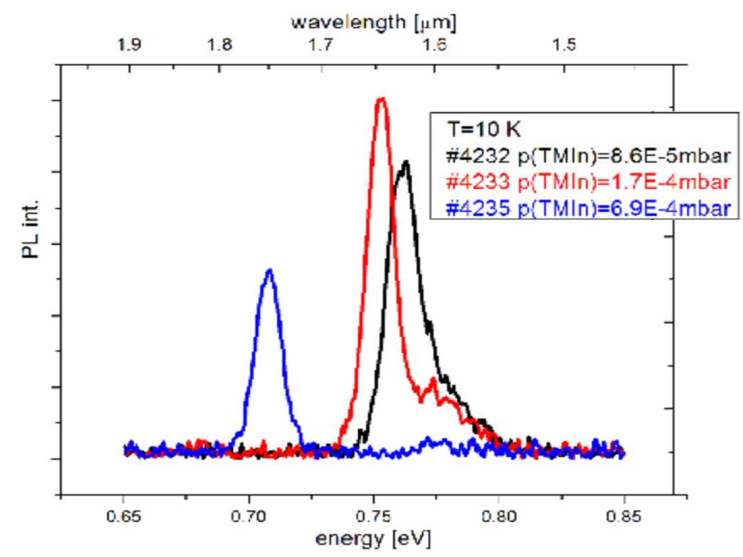

Fig. 3. Photoluminescence spectra of $\operatorname{In}_{x} \mathrm{Ga}_{1-x} \mathrm{Sb} /$ GaSb layers for three values of TMIn precursor partial pressure during epitaxial process: $8.6 \times 10^{-5}$ mbar, $1.7 \times 10^{-4}$ mbar and $6.9 \times 10^{-4}$ mbar.

The band-gap energy of antimonide layers was also observed by photoreflectance spectroscopy. Spectrum for InGaAsSb/GaSb heterostructure is presented in Fig. 4. These are visible are two transitions: one occurring in the GaSb substrate, second - in the epi-layer. Knowledge of energy gap derived from photoreflectance or photoluminescence together with the lattice constant from XRD allowed to resolve quaternary composition, which in case of sample from Fig. 4 was assumed to be $\mathrm{In}_{0.06} \mathrm{Ga}_{0.94} \mathrm{As}_{0.04} \mathrm{Sb}_{0.96}$.

Electrical measurements, because of the lack of GaSb semi-insulating (SI) substrates, were provided with specially prepared samples grown on SI GaAs wafers. Undoped InGaSb layers indicated typically $p$-type concentration in the range of $5 \times 10^{16} \mathrm{~cm}^{-3}$ with mobility $170 \mathrm{~cm}^{2} /(\mathrm{V} \mathrm{s})$. These values could be affected by substrate/layer interface. Such antimonide layers grown on GaAs substrates were also used in absorption spectroscopy for additional determination of bandgap energy. 


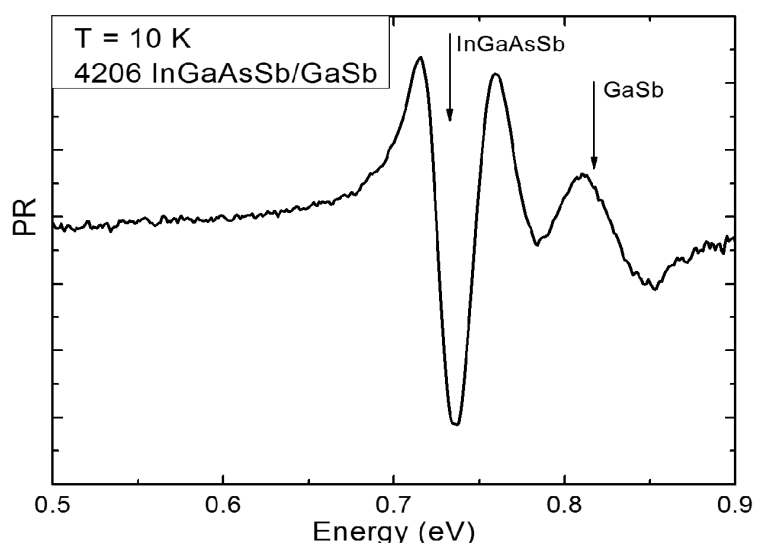

Fig. 4. Photoreflectance spectrum of InGaAsSb/GaSb heterostructure.

The indium contents derived from absorption edge of InGaSb/GaAs were in good accordance with values obtained from XRD.

The SIMS technique has shown increased levels of carbon and oxygen contaminations, as compared to substrate values. For carbon it was usually 1.5 orders, in case of oxygen 2-3 orders of magnitude. These levels did not highly depend on compound composition factors, however oxygen level should further grow if aluminium-containing antimonides were considered.

TEM imaging taken for GaSb/InGaSb/GaAsSb/ InGaAsSb/GaSb heterostructure did not show any distinct structural defects.

\section{Conclusions}

Developed MOCVD epitaxy of several antimonides on GaSb allowed to produce good quality layers, which can be considered for application in optoelectronic devices. Some attributes, like properties exhibited for selected structures by microscopy and XRD, are already satisfied, other - need to be improved. The issue for solution is the oxygen contamination - main factor causing MBE to be currently more successful in the field.

\section{Acknowledgments}

The research was financed from the project "Advanced technologies for semiconductor infrared optoelectronics", PBZ-MniSW 02/I/2007.

\section{References}

[1] P.S. Dutta, H.L. Bhat, Vikram Kumar, J. Appl. Phys. 81, 5821 (1997).

[2] F. Dimroth, C. Agert, A.W. Bett, J. Cryst. Growth 248, 265 (2003).

[3] A. Aardvark, N.J. Mason, P.J. Walker, Prog. Crystal Growth Charact. 35, 207 (1997).

[4] R.M. Biefeld, Mater. Sci. Eng. R 36, 105 (2002).

[5] C.A. Wang, J. Cryst. Growth 272, 664 (2004). 